\title{
On the Computation of the Direct Kinematics of Parallel Spherical Mechanisms Using Bernstein Polynomials
}

\author{
Carlos Bombín, Lluís Ros, and Federico Thomas \\ Institut de Robòtica i Informàtica Industrial (CSIC - UPC) \\ Gran Capità 2-4, 2 planta, 08034 Barcelona. SPAIN \\ \{cbombin, llros, fthomas\}@iri.upc.es
}

\begin{abstract}
Solving the direct kinematics of parallel spherical mechanisms with $l$ legs boils down to solving systems of $l-1$ second-order multinomials. This paper presents a recurrent expression for the control points of these multinomials when expressed in Bernstein form. This result allows us to propose a technique for solving the direct kinematics of these mechanisms that takes advantage of the subdivision and convex hull properties of polynomials in Bernstein form. Contrary to other numerical approaches, the one presented here is clearly less involved and, although it can be classified within the same category as interval-based techniques, it does not require any interval arithmetic computation.
\end{abstract}

\section{Introduction}

Parallel manipulators are closed-chain mechanisms with one or more loops where only a certain number of pairs are actively controlled. Fully parallel mechanisms, in particular, feature two rigid bodies, termed base and platform, connected by a set of legs. Position analysis of a parallel manipulator involves a direct and an inverse kinematic problem. In general, the inverse problem is trivial, since it asks for the legs' configurations when the position and orientation of the platform are given with respect to the base. On the contrary, the direct problem, which calls for the position and orientation of the platform when the configurations of the actively controlled pairs are given, is a difficult problem for which no general procedure has been found yet and for which closed-form solutions are only available for certain architectures, sometimes satisfying a number of geometric conditions [8].

The different architectures for parallel mechanisms can be analitically studied by a customized strategy that can be summarized as follows. First, the configuration of the platform with respect to the base is parameterized, so that a closure system with a reduced number of equations and unknowns can be written. Second, using a suitable elimination procedure a final polynomial equation in only one unknown is obtained. Unfortunately, both steps heavily rely on the geometric intuition of the researcher. The roots of the final polynomial lead to the sought solutions by substitution. Consequently, the order of the polynomial equation does not necessarily represent the number of solutions in the real field and the real roots do not necessarily correspond to configurations within the mechanical limits of the mechanism under consideration. However, despite these drawbacks, this has been the usual approach to the problem and it has triggered the hunt for the lowest order polynomial associated with each architecture.

In this context, current numerical methods have been laid aside because of their proved difficulty to find all solutions. Our group has been working on the application of interval-based techniques to provide a way around this difficulty. In particular, we have applied interval extensions of Newton methods, coupled with bisection to ensure convergence, to solve the inverse kinematic problem of serial manipulators directly from their Denavit-Hartenberg parameters [1, 2]. The rather difficult generalization of our algorithm to multiple loops and its complex implementation among other drawbacks - led us to explore other alternatives. Here we develop a general technique to numerically solve systems of spherical kinematic constraints directly from the Denavit-Hartenberg parameters of the involved kinematic chains that relies on the properties of polynomials in Bernstein form. 

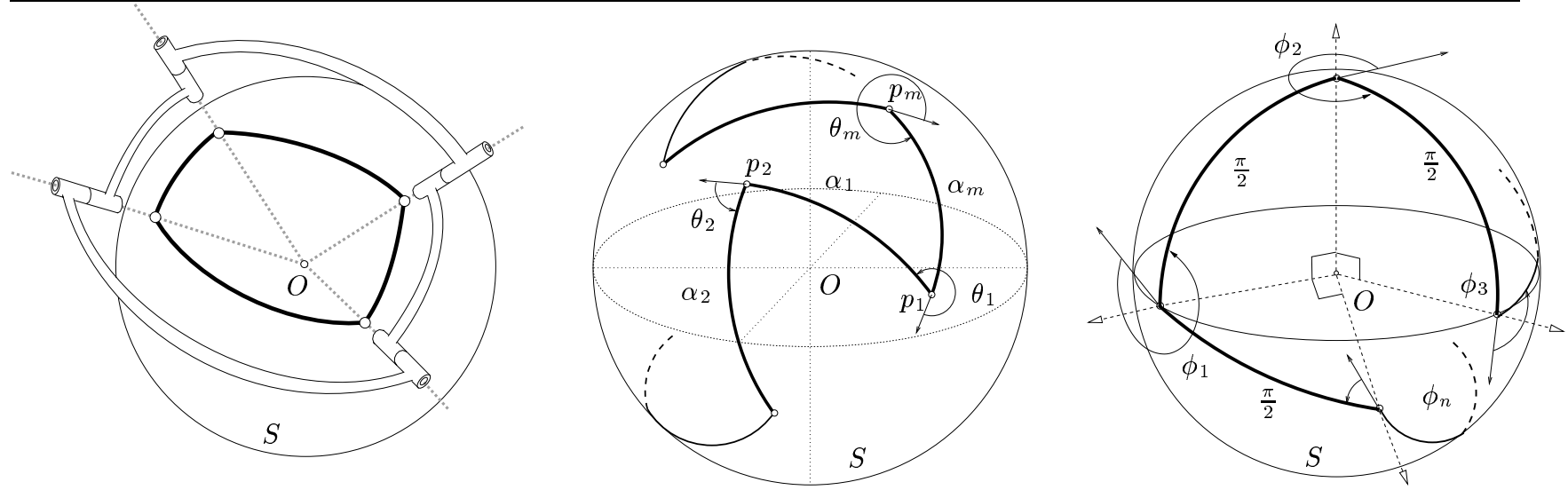

Figure 1: Left: The spherical polygon associated with a spherical 4-bar linkage. Center and right: a spherical polygon before and after normalization.

We begin by introducing, in Section 2, some basic concepts and notations, upon which a standard formulation of spherical problems is developed. Section 3 states our main theoretical results that provide the basis for the application of a subdivisionminimization strategy, described in Section 4, to solve the direct kinematics problem on arbitrary parallel spherical mechanisms. We conclude in Section 5.

\section{Spherical polygons and closure equa- tions}

A mechanism is called spherical if each of its links rotates about a same fixed point, say $O$. Thus, trajectories of points in each link lie on concentric spheres with $O$ as the center. Only the revolute joint is compatible with this movement and its axis must pass through the fixed point. For convenience, we may think that all revolute joints lie on the same sphere $S$, and that the links are portions of great circles on $S$ (fig. 1, left). This is how every closed chain of a parallel spherical mechanism defines a spherical polygon on $S$. For each of these polygons we can derive a closure equation, as explained next.

If we assign a circulating direction to the sides of the polygon, the exterior angle between two adjacent sides is defined as the angle measured from the prolongation beyond the common vertex of the first side to the second side. Next, consider a spherical polygon with vertices $p_{1}, \ldots, p_{m}$, sides $\alpha_{1}, \ldots, \alpha_{m}$, and exterior angles $\theta_{1}, \ldots, \theta_{m}$ (fig. 1, center). For each side, we define its pole as the point of the sphere lying on a line through the center $O$, perpendicular to the plane of the side. Now, take a system of coordinate axes centered at $O$ with the $x$-axis passing through $p_{1}$ and its $z$-axis passing through the pole of side $\alpha_{1}$. A rotation of $\alpha_{1}$ radians about the $z$-axis will move the $x$-axis along the side $\alpha_{1}$ till vertex $p_{2}$. Next, a rotation of angle $\theta_{2}$ about the new $x$-axis will make the $z$-axis pass through the pole of $\alpha_{2}$. Going on in this way all around the polygon, the $x$-axis will return to $p_{1}$ and the coordinate system will end up being in its original position. This may be expressed by means of a rotation equation stating that the composition of the successive rotations equals the identity transformation, that is,

$$
\prod_{i=1}^{m} \mathbf{R x}\left(\theta_{i}\right) \mathbf{R} \mathbf{z}\left(\alpha_{i}\right)=\mathbf{I} .
$$

where $\mathbf{R x}(\cdot)$ and $\mathbf{R z}(\cdot)$ stand for $3 \times 3$ orthogonal matrices representing rotations about the $x$ and $z$ axes, respectively, in the amounts given by their arguments. Alternatively to this formulation, we can write

$$
\prod_{i=1}^{m} \mathbf{R x}\left(\theta_{i}+\pi\right) \mathbf{R} \mathbf{z}(\pi / 2) \mathbf{R} \mathbf{x}\left(\alpha_{i}+\pi\right) \mathbf{R} \mathbf{z}(\pi / 2)=\mathbf{I},
$$

which corresponds to the closure equation of a spherical polygon where all its sides have length $\pi / 2$, thus involving variable rotations about the same axis (fig. 1, right). This normalization will simplify our further algebraic manipulations. Then, Equation (1) can be rewritten as:

$$
\mathbf{F}_{n}(\Phi)=\prod_{i=1}^{n} \mathbf{R x}\left(\phi_{i}\right) \mathbf{Z}=\mathbf{I},
$$

where $\mathbf{Z}=\mathbf{R z}(\pi / 2), n=2 m, \Phi=\left(\phi_{1}, \ldots, \phi_{n}\right)$, and

$$
\phi_{i}=\left\{\begin{array}{lll}
\theta_{\frac{i+1}{2}}+\pi, & i \text { even } \\
\alpha_{\frac{i}{2}}+\pi, & i \text { odd }
\end{array}\right.
$$



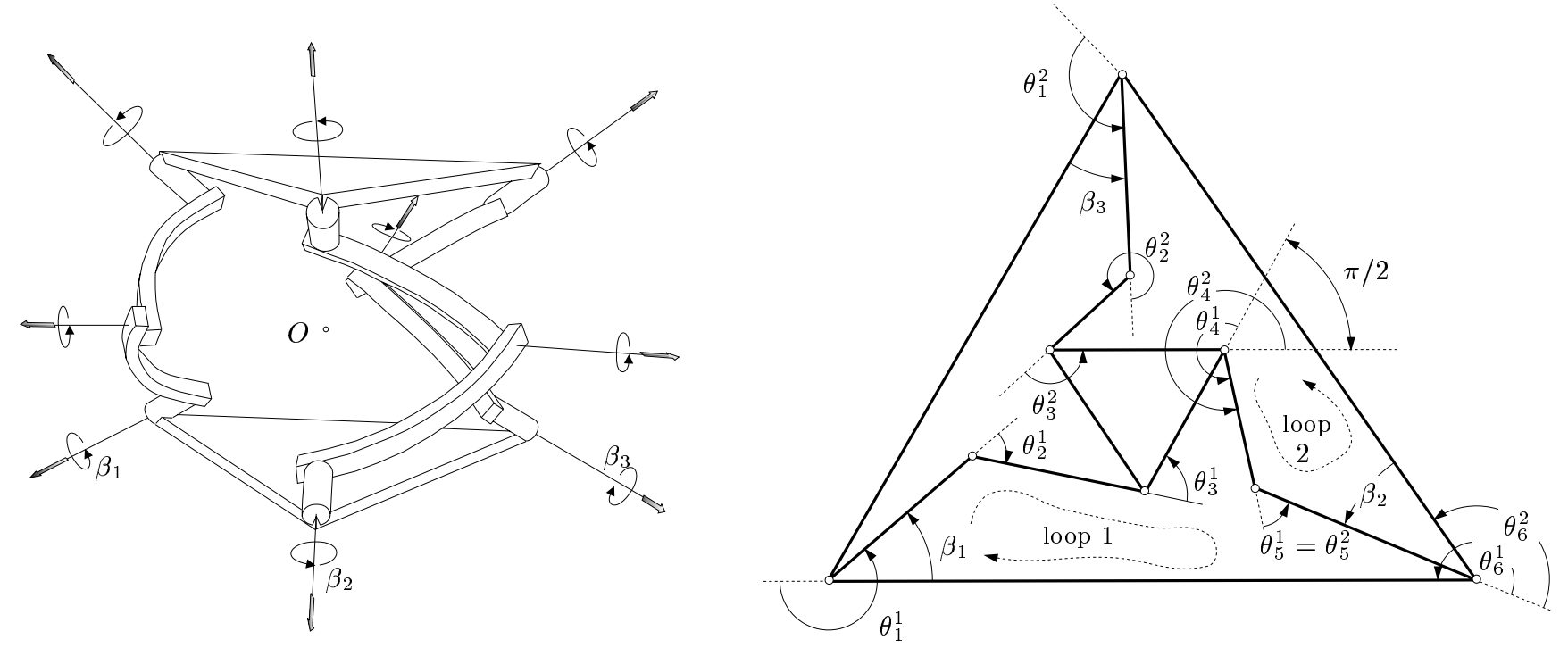

Figure 2: The Gosselin platform (left), and the spherical polygons it defines (right). While the $\beta_{i}$ 's are actively controlled, all other variables are passive revolute joints.

The solution set to $\mathbf{F}_{n}(\Phi)=\mathbf{I}$, a system of nine trigonometric polynomials in $n$ variables, has been geometrically and topologically characterized in [3].

\subsection{An example}

Consider the spherical parallel manipulator shown in fig. 2, sometimes called the Gosselin platform. It consists of a platform connected to a fixed base via three kinematic chains, each composed of two intermediate links and three revolute joints. Only the revolute joints connected to the base are actuated. When the link angles and the angles between the revolute axes on the base and on the platform are all set to $\pi / 2$, a special geometry is obtained for which a closed-form solution has been derived [6]. It has been shown that the direct kinematic problem of three-degree-of-freedom parallel manipulators has a maximum of 8 solutions [5, Section 3]. The closed-form solution of the Gosselin platform accounts for 8 solutions, as expected, but 4 of them are singular configurations in which the actuators can be moved arbitrarily without affecting the pose of the platform. In practice, such singular configurations should not be inside the workspace of the manipulator, which can be accomplished by limiting the range of motion of the active joints.

Two equations are sufficient to study the kinematics of this manipulator, corresponding to the two loops indicated in fig. 2, right. With the indicated angles, these equations are:

$$
\begin{array}{ll}
\prod_{i=1}^{6} \mathbf{R x}\left(\theta_{i}^{1}\right) \mathbf{Z}=\mathbf{I}, & \text { for loop } 1 \\
\prod_{i=1}^{6} \mathbf{R x}\left(\theta_{i}^{2}\right) \mathbf{Z}=\mathbf{I}, & \text { for loop } 2 .
\end{array}
$$

Note that, due to the special geometry of the manipulator, these are already in the normal form of Equation (2). The angles of the actuators directly determine four variables in the previous equations, because $\theta_{1}^{1}=\pi+\beta_{1}, \theta_{6}^{1}=\frac{\pi}{2}-\beta_{2}, \theta_{6}^{2}=\pi-\beta_{2}$ and $\theta_{1}^{2}=\frac{3 \pi}{2}+\beta_{3}$. Moreover, from fig. 2 we see that two variables in one loop are related to others in the second:

$$
\theta_{4}^{2}=\theta_{4}^{1}+\pi / 2, \quad \theta_{5}^{2}=\theta_{5}^{1} .
$$

Thus, substituting (5) in Equations (3) and (4), and using the fact that

$$
\mathbf{R x}\left(\phi_{1}+\phi_{2}\right)=\mathbf{R x}\left(\phi_{2}+\pi\right) \mathbf{Z} \cdot \mathbf{R x}(\pi) \mathbf{Z} \cdot \mathbf{R x}\left(\phi_{1}\right),
$$

we finally get two equations involving six variables,

$$
\begin{aligned}
& \mathbf{R x}\left(\pi+\beta_{1}\right) \mathbf{Z} \cdot \mathbf{R x}\left(\theta_{2}^{1}\right) \mathbf{Z} \cdot \mathbf{R x}\left(\theta_{3}^{1}\right) \mathbf{Z} \cdot \\
& \quad \cdot \mathbf{R x}\left(\theta_{4}^{1}\right) \mathbf{Z} \cdot \mathbf{R x}\left(\theta_{5}^{1}\right) \mathbf{Z} \cdot \mathbf{R x}\left(\frac{\pi}{2}-\beta_{2}\right) \mathbf{Z}=\mathbf{I} \\
& \mathbf{R x}\left(\pi-\beta_{2}\right) \mathbf{Z} \cdot \mathbf{R x}\left(\theta_{2}^{2}\right) \mathbf{Z} \cdot \mathbf{R x}\left(\theta_{3}^{2}\right) \mathbf{Z} \cdot \mathbf{R x}\left(-\frac{\pi}{2}\right) \mathbf{Z} \cdot \\
& \quad \cdot \mathbf{R x}(\pi) \mathbf{Z} \cdot \mathbf{R x}\left(\theta_{4}^{1}\right) \mathbf{Z} \cdot \mathbf{R x}\left(\theta_{5}^{1}\right) \mathbf{Z} \cdot \mathbf{R x}\left(\frac{3 \pi}{2}+\beta_{3}\right) \mathbf{Z}=\mathbf{I} .
\end{aligned}
$$




\subsection{Reduction to scalar equations}

Due to the fact that $\mathbf{F}_{n}(\Phi)=\left[f_{i j}^{n}(\Phi)\right]_{1 \leq i, j \leq 3}$ is a product of orthogonal matrices equated to the identity, it can be reduced to the following single trigonometric equation:

$$
f_{11}^{n}(\Phi)+f_{22}^{n}(\Phi)-2=0 .
$$

Now, $f_{i j}^{n}(\Phi)$ can be converted into a rational polynomial $\overline{\overline{f_{i j}^{n}}}(\mathbf{t})$ in a new variable, $\mathbf{t}=\left(t_{1}, \ldots, t_{n}\right)$, using the tangent-half-angle substitution, that is, by introducing the substitutions $\sin \left(\phi_{i}\right)=\frac{2 t_{i}}{1+t_{i}^{2}}$ and $\cos \left(\phi_{i}\right)=$ $\frac{1-t_{i}^{2}}{1+t_{i}^{2}}$. Then, if we multiply the resulting rational polynomials by $q_{n}(\mathbf{t})=\prod_{i=1}^{n}\left(1+t_{i}^{2}\right)$, we obtain the polynomials $\overline{f_{i j}^{n}}(\mathbf{t})=q_{n}(\mathbf{t}) \overline{\overline{f_{i j}^{n}}}(\mathbf{t})$ (we adhere to the notation introduced in [7]). Therefore, Equation (2) can be expressed as:

$$
\overline{f_{11}^{n}}(\mathbf{t})+\overline{f_{22}^{n}}(\mathbf{t})-2 q_{n}(\mathbf{t})=0 .
$$

For an arbitrary function in $n$ variables, say $g(\mathbf{t})=g\left(t_{1}, \ldots, t_{n}\right)$, we define $g^{\sigma}(\mathbf{t})=g\left(\mathbf{t}^{\sigma}\right)=$ $g\left(t_{n}, t_{1}, \ldots, t_{n-1}\right)$. Then, if we denote $\mathbf{F}_{n}^{\sigma}(\Phi)=$ $\left[f_{i j}^{n \sigma}(\Phi)\right]_{1 \leq i, j \leq 3}$, we have that

$$
\mathbf{F}_{n}^{\sigma}(\Phi)=\left(\mathbf{R x}\left(\phi_{n}\right) \mathbf{Z}\right) \mathbf{F}_{n}(\Phi)\left(\mathbf{R x}\left(\phi_{n}\right) \mathbf{Z}\right)^{t} .
$$

Now, the element $(1,1)$ of $\mathbf{F}_{n}^{\sigma}$, i.e. $f_{11}^{n \sigma}(\Phi)$, is equal to the element $(1,1)$ of the product $\left(\mathbf{R x}\left(\phi_{n}\right) \mathbf{Z}\right) \mathbf{F}_{n}(\Phi)\left(\mathbf{R x}\left(\phi_{n}\right) \mathbf{Z}\right)^{t}$, that can be checked to be $f_{22}^{n}(\Phi)$. In other words, Equation (8) can be rewritten as:

$$
\overline{f_{11}^{n}}(\mathbf{t})+\overline{f_{11}^{n}}\left(\mathbf{t}^{\sigma}\right)-2 q_{n}(\mathbf{t})=0 .
$$

Once the ranges of motions for the passive joints are given, the singularity of the tangent-half-angle substitution at $\pi$ can be avoided by shifting them a given amount using the relation (6), provided that these ranges are lower than $2 \pi$.

\section{The closure equations in Bernstein form}

Let $M=\left(m_{1}, \ldots, m_{n}\right)$ and $I=\left(i_{1}, \ldots, i_{n}\right)$. The notation $0 \leq I \leq M$ indicates that $0 \leq i_{k} \leq m_{k}$, for $k=1, \ldots, n$. It is well known that $\mathbb{R}_{M}[\mathbf{t}]$, the set of polynomials in the variables $t_{1}, \ldots, t_{n}$ of degree $\leq m_{i}$ in $t_{i}$, is a vector space. Also, the Bernstein multinomials $\left\{B_{I, M}(\mathbf{t})\right\}_{\mathbf{0} \leq I \leq M}$ defined as $B_{I, M}(\mathbf{t})=$ $b_{i_{1}, m_{1}}\left(t_{1}\right) \cdots b_{i_{n}, m_{n}}\left(t_{n}\right)$ (where $b_{i, m}$ denotes the $i$ th Bernstein polynomial of degree $m$ ) forms a basis of
$\mathbb{R}_{M}[\mathbf{t}]$, called the multivariate Bernstein basis. Therefore, any polynomial $f(\mathbf{t}) \in \mathbb{R}_{M}[\mathbf{t}]$ can be written as $f(\mathbf{t})=\sum_{I=0}^{M} c_{I}(f) B_{I, M}(\mathbf{t})$. (For simplicity, we write $B_{I}(\mathbf{t})$ instead of $B_{I, M}(\mathbf{t})$.) This expression is the Bernstein form of $f(\mathbf{t})$ and the coefficients $c_{I}(f)$ are called its control points.

In our case, we are interested in the Bernstein form of the polynomial $f=\overline{f_{11}^{n}}+{\overline{f_{11}^{n}}}^{\sigma}-2 q_{n} \in \mathbb{R}_{M}[\mathbf{t}]$, where $M=(2, \ldots, 2) \in \mathbb{R}^{n}$. It is easy to prove that $c_{I}(f)=$ $c_{I}\left(\overline{f_{11}^{n}}\right)+c_{I}\left(\bar{f}_{11}^{n} \sigma\right)-2 c_{I}\left(q_{n}\right)$.

Proposition 1. The control points of $q_{n}(\mathbf{t})$ are $c_{I}\left(q_{n}\right)=2^{\chi(I)}$, where $\chi(I)$ is the number of elements of $I$ equal to 2.

Proof. It can be checked that $q\left(t_{i}\right)=$ $b_{0,2}\left(t_{i}\right)+b_{1,2}\left(t_{i}\right)+2 b_{2,2}\left(t_{i}\right), \quad$ therefore the polynomial $q_{n}$ can be expressed as: $q_{n}(\mathbf{t})=\prod_{i=1}^{n}\left[b_{0,2}\left(t_{i}\right)+b_{1,2}\left(t_{i}\right)+2 b_{2,2}\left(t_{i}\right)\right]=$ $\sum_{I=\mathbf{0}}^{M} 2^{\chi(I)} B_{I}(\mathbf{t})$.

Since it can be easily shown to be $c_{I}\left({\overline{f_{11}^{n}}}^{\sigma}\right)=$ $c_{I^{\sigma}}\left(\overline{f_{11}^{n}}\right)$, where $I^{\sigma}=\left(i_{n}, i_{1}, \ldots, i_{n-1}\right)$, we only have to calculate the control points $c_{I}\left(\overline{f_{11}^{n}}\right)$, but first we need the following proposition:

Proposition 2. $f_{11}^{n}$ and $f_{13}^{n}$ satisfy the recursion:

$$
\begin{aligned}
f_{11}^{n}\left(\phi_{1}, \ldots, \phi_{n}\right)= & -f_{11}^{n-2}\left(\phi_{1}, \ldots, \phi_{n-2}\right) \cos \left(\phi_{n}\right)+ \\
& +f_{13}^{n-1}\left(\phi_{1}, \ldots, \phi_{n-1}\right) \sin \left(\phi_{n}\right) \\
f_{13}^{n}\left(\phi_{1}, \ldots, \phi_{n}\right)= & f_{11}^{n-2}\left(\phi_{1}, \ldots, \phi_{n-2}\right) \sin \left(\phi_{n}\right)+ \\
& +f_{13}^{n-1}\left(\phi_{1}, \ldots, \phi_{n-1}\right) \cos \left(\phi_{n}\right) .
\end{aligned}
$$

Proof. The proof can be carried out by elementary algebraic manipulations of the matrix products in (2).

Corollary 1. $\overline{f_{11}^{n}}$ and $\overline{f_{13}^{n}}$ satisfy the recursion:

$$
\begin{array}{r}
\overline{f_{11}^{n}}\left(t_{1}, \ldots, t_{n}\right)=\overline{f_{11}^{n-2}}\left(t_{1}, \ldots, t_{n-2}\right) g_{1}\left(t_{n-1}, t_{n}\right)+ \\
+\overline{f_{13}^{n-1}}\left(t_{1}, \ldots, t_{n-1}\right) h_{1}\left(t_{n}\right) \\
\overline{f_{13}^{n}}\left(t_{1}, \ldots, t_{n}\right)=\overline{f_{11}^{n-2}}\left(t_{1}, \ldots, t_{n-2}\right) g_{2}\left(t_{n-1}, t_{n}\right)- \\
\quad-\overline{f_{13}^{n-1}}\left(t_{1}, \ldots, t_{n-1}\right) h_{2}\left(t_{n}\right),
\end{array}
$$

where, $h_{1}\left(t_{n}\right)=2 t_{n}, h_{2}\left(t_{n}\right)=t_{n}^{2}-1, g_{1}\left(t_{n-1}, t_{n}\right)=$ $\left(t_{n-1}^{2}+1\right) h_{2}\left(t_{n}\right)$, and $g_{2}\left(t_{n-1}, t_{n}\right)=\left(t_{n-1}^{2}+1\right) h_{1}\left(t_{n}\right)$.

Corollary 2. The control points of $\overline{f_{11}^{n}}$ and $\overline{f_{13}^{n}}$ satisfy the recursion:

$$
\begin{aligned}
c_{\left(i_{1}, \ldots, i_{n}\right)}\left(\overline{f_{11}^{n}}\right)= & c_{\left(i_{1}, \ldots, i_{n-2}\right)}\left(\overline{f_{11}^{n-2}}\right) c_{\left(i_{n-1}, i_{n}\right)}\left(g_{1}\right)+ \\
& c_{\left(i_{1}, \ldots, i_{n-1}\right)}\left(\overline{f_{13}^{n-1}}\right) c_{i_{n}}\left(h_{1}\right) \\
c_{\left(i_{1}, \ldots, i_{n}\right)}\left(\overline{f_{13}^{n}}\right)= & c_{\left(i_{1}, \ldots, i_{n-2}\right)}\left(\overline{f_{11}^{n-2}}\right) c_{\left(i_{n-1}, i_{n}\right)}\left(g_{2}\right)- \\
& c_{\left(i_{1}, \ldots, i_{n-1}\right)}\left(\overline{f_{13}^{n-1}}\right) c_{i_{n}}\left(h_{2}\right),
\end{aligned}
$$


where the control points of $h_{1}, h_{2}, g_{1}$, and $g_{2}$ different from zero are:

$$
\begin{array}{r}
c_{(0,0)}\left(g_{1}\right)=c_{(0,1)}\left(g_{1}\right)=c_{(1,0)}\left(g_{1}\right)=c_{(1,1)}\left(g_{1}\right)=-1, \\
c_{(2,0)}\left(g_{1}\right)=c_{(2,1)}\left(g_{1}\right)=-2, \\
c_{(0,1)}\left(g_{2}\right)=c_{(1,1)}\left(g_{2}\right)=1, \\
c_{(0,2)}\left(g_{2}\right)=c_{(1,2)}\left(g_{2}\right)=c_{(2,1)}\left(g_{2}\right)=2, \\
c_{(2,2)}\left(g_{2}\right)=4, \\
c_{1}\left(h_{1}\right)=1, \\
c_{2}\left(h_{1}\right)=2, \\
c_{0}\left(h_{2}\right)=-1, \\
c_{1}\left(h_{2}\right)=-1 .
\end{array}
$$

Finally, we have that the Bernstein form of Equation (9) is:

$$
\sum_{I=\mathbf{0}}^{M}\left[c_{I}\left(\overline{f_{11}^{n}}\right)+c_{I} \sigma\left(\overline{f_{11}^{n}}\right)-2^{\chi(I)+1}\right] B_{I}(\mathbf{t})=0,
$$

where $c_{I}\left(\overline{f_{11}^{n}}\right)$ and $c_{I^{\sigma}}\left(\overline{f_{11}^{n}}\right)$ can be computed using Corollary 2.

So far, for simplicity, we have treated all $\phi_{i}$ as variables, thus yielding $3^{n}$ control points in Equation (10). In practice though, many variables correspond to known angles of the mechanism and hence only a reduced set of control points is actually needed. For example, for the Gosselin platform each equation has four variables. Then, we have to compute $3^{4}=81$ control points to write term in the form of Equation (10). That is, altogether, 162 control points will fully describe the mechanism.

\section{A subdivision-minimization strategy}

Remind that a closure equation is obtained for every couple of legs. Then, note that the number of independent closure equations we can derive is $l-1$ for a mechanism with $l$ legs. Nevertheless, to simplify the presentation, let us assume that we are working with only one closure equation, i.e., we need to compute the solutions to just one equation of the form given by (9). The generalization will appear obvious at the end.

We are going to apply a method that allows searching for those roots of a Bernstein-form polynomial in $n$ variables that lie in the unit box $[0,1]^{n}$ of $\mathbb{R}^{n}$. Since the variables $t_{i}$ in Equation (10) take values in their range, we first apply an affine parameter transformation to it so that the initial box is converted into the unit box. This scaling yields a new polynomial in Bernstein form with a new set of control points [4, Sec. 15.7]. Let us write it as $f(\mathbf{x})=$ $\sum_{I=\mathbf{0}}^{M} w_{I} B_{I}(\mathbf{x})$, where $\mathbf{x}$ stands for $\left(x_{1}, \ldots, x_{n}\right)$, and let us construct the function $F: \mathbb{R}^{n} \longrightarrow \mathbb{R}^{n+1}$ defined as $F(\mathbf{x})=(\mathbf{x}, f(\mathbf{x}))$. Trivially, finding the roots of $f(\mathbf{x})$ is equivalent to detecting all points of the form $(\mathbf{x}, 0)$ in the graph of $F(\mathbf{x})$. However, the latter formulation is advantageous. First, the graph of $F(\mathbf{x})$ is an algebraic variety in $\mathbb{R}^{n+1}$ whose points can be parameterized with polynomials in Bernstein form as

$$
F(\mathbf{x})=\sum_{I=0}^{M} v_{I} B_{I}(\mathbf{x}),
$$

where $v_{I}=\left(i_{1} / m_{1}, i_{2} / m_{2}, \ldots, i_{n} / m_{n}, w_{I}\right)$, which are called the control points of $F(\mathbf{x})[9]$.

Now, the root-finding procedure can make use of two important properties of the Bernstein form of $F(\mathbf{x})$. The first one is the so-called convex hull property: when $\mathbf{x} \in[0,1]^{n}, F(\mathbf{x})$ is totally contained within the convex hull of its control points $v_{I}$. This follows immediately from the values taken by the Bernstein polynomials $B_{I}$ in the unit box. They all are nonnegative and sum to 1 [4], and hence the linear combination of control points $v_{I}$ in $\sum_{I=0}^{M} v_{I} B_{I}(\mathbf{x})$ is actually a convex combination when $\mathbf{x} \in[0,1]^{n}$. The second property is subdivision: if we are interested in the values that $F(\mathbf{x})$ takes within a sub-box of $[0,1]^{n}$, say $\mathcal{B}=\left[a_{1}, b_{1}\right] \times\left[a_{2}, b_{2}\right] \times \ldots \times\left[a_{n}, b_{n}\right]$, with $0 \leq a_{i}, b_{i} \leq 1$, then it is possible to apply an affine parameter transformation $x_{i}=a_{i}+u_{i}\left(b_{i}-a_{i}\right), i=1, \ldots, n$, to scale $\mathcal{B}$ to the unit box and rewrite $F$ in Bernstein form in terms of the new parameters $u_{i}$, with new control points. The important point here is that, after the scaling, the new control points for $F$ can be directly obtained from the control points of the initial $F(\mathbf{x})$, and they will be closer to the graph of $F$ than the previous ones [4, Sec. 15.7]. These considerations permit the following procedure to find all the roots of Equation (10).

1. Compute the control points $v_{I}$ of $F(\mathbf{x})$. Start with the box $\mathcal{B}=[0,1]^{n}$.

2. Using the convex hull property, find a sub-box $\mathcal{B}^{\prime}$ of $\mathcal{B}$ that contains all the solutions of $F(\mathbf{x})=(\mathbf{x}, 0)$ (see the details below). If there is no such sub-box (i.e., $\mathcal{B}$ contains no solution), set $\mathcal{B}^{\prime}=\varnothing$.

3. If $\mathcal{B}^{\prime} \neq \varnothing$, see if it is sufficiently small. If so, conclude that there is a root inside and return $\mathcal{B}^{\prime}$; otherwise split $\mathcal{B}^{\prime}$ into some number of equally sized smaller boxes, scale these boxes back to $[0,1]^{n}$ using the subdivision property for $F$, and recursively call step 2 once for each new smaller box.

It remains to see how step 2 is performed. Let $\mathcal{C}$ denote the convex hull of the control points $v_{I}$, and let $\mathcal{R}$ be the region of intersection of $\mathcal{C}$ with the hyperplane $x_{n+1}=0$. Then, we define $\mathcal{B}^{\prime}$ as the smallest rectangular box enclosing $\mathcal{R}$. Although the explicit computation of $\mathcal{R}$ is a complex and time-consuming 
task, it is not necessary to carry it out explicitly if all we need is just a bounding box for it. Indeed, $\mathcal{R}$ can be described with a set of linear equalities and inequalities as follows. Since a point in $\mathcal{R}$ is of the form $(\mathbf{x}, 0)$ and it must be a convex combination of the control points $v_{I}$, there must be coefficients $c_{I} \in \mathbb{R}$ such that

$$
(\mathbf{x}, 0)=\sum_{I=\mathbf{0}}^{M} c_{I} v_{I}, \quad c_{I} \geq 0 \quad \forall I, \quad \text { and } \sum_{I=\mathbf{0}}^{M} c_{I}=1 .
$$

Now, to obtain the bounds of the box $\mathcal{B}^{\prime}$ we simply need to maximize and minimize $x_{i}$, for $i=1, \ldots, n$ subject to the constraints in Equations (12). These optimizations are linear programming problems and, hence, they can be efficiently solved with the simplex algorithm.

The above algorithm has been proven to terminate in all cases. Moreover, if there is a finite number of roots, then it returns a box enclosing each of them that is smaller than a user-specified tolerance. If the number of roots is infinite, the algorithm also terminates, providing a discretization of the solution space in a number of small boxes enclosing it. Additionally, the algorithm has the good property of being quadratically convergent to the roots. See [9] for details on all these facts.

This process is straightforwardly generalizable to mechanisms with several loops as follows. For each loop $j$ we will have one closure equation $f_{j}(\mathbf{x})=0$, with its corresponding function $F_{j}(\mathbf{x})=\left(x, f_{j}(\mathbf{x})\right)$ written as in Equation (11), and an associated convex hull $\mathcal{C}_{j}$, wrapping the control points of $F_{j}(\mathbf{x})$. Note that the common solutions to the whole system of equations necessarily lie in the intersection of all $\mathcal{C}_{j}$ 's, on $x_{n+1}=0$. This just introduces a slight modification in step 2 of the previous algorithm: as before, $\mathcal{B}^{\prime}$ is computed from $\mathcal{B}$ by minimizing each $x_{i}, i=1, \ldots, n$, but now subject to all linear constraints in (12), associated with all $\mathcal{C}_{j}$ 's.

\section{Conclusions}

We have presented an algorithm for solving the direct kinematics of parallel spherical mechanisms using a technique that takes advantage of the subdivision and convex-hull properties of the polynomials in Bernstein form, a technique developed in the context of Computer Graphics applications. This has been possible thanks to the recursion found for the control points of the closure equations.

We have used a tangent-half-angle substitution. This is probably the worst possible algebraic parameterization of the unit circle. Alternatively, it would be possible to apply the substitutions $x_{i}=\sin \phi_{i}$ and $y_{i}=\cos \phi_{i}$. Then, the equation $x_{i}^{2}+y_{i}^{2}=1$ should be included in the resulting system of equations. This would have at least two main advantages: the singularity at $\phi_{i}=\pi$, inherent to the tangent-half-angle substitution, would be avoided; and the set of control points would be greatly simplied because the closure equations would become linear in all the variables. These points concentrate our current efforts.

\section{Acknowledgments}

The authors would like to acknowledge the financial support of the Spanish CICYT under contract TAP991086-C03-01.

\section{References}

[1] A. Castellet, Solving inverse kinematics problems using an interval method, Ph.D. Thesis, Polytechnical University of Catalonia. Available at http://wwwiri.upc.es/people/thomas/students.html

[2] A. Castellet and F. Thomas, "Towards an efficient interval method for solving inverse kinematic problems," IEEE Int. Conf. on Robotics and Automation, Vol. IV, pp. 3615-3620, 1997.

[3] A. Castellet and F. Thomas, "The self-motion manifold of the orthogonal spherical mechanism," Mechanisms and Machine Theory, Vol. 34, No. 1, pp. 59-88, 1999.

[4] G. Farin, Curves and surfaces for computer-aided geometric design - A practical guide. 2nd edition. Academic Press, 1990.

[5] C.M. Gosselin, J. Sefrioui, and M.J. Richard, "On the direct kinematics of spherical three-degree-of-freedom parallel manipulators of general architecture," ASME Journal of Mechanical Design, Vol. 116, No. 2, pp. 594-598, 1994.

[6] C.M. Gosselin, and M. Gagné, "A closed-form solution for the direct kinematics of a special class of spherical three-degree-of-freedom parallel manipulators," in Computational Kinematics, J.-P. Merlet and B. Ravani (eds.), pp. 231-240, Kluwer Academic Publishers, 1995.

[7] P. Kovács and G. Hommel, "On the tangent-halfangle substitution," in Computational Kinematics, J. Angeles (editor), pp. 27-39, Kluwer Academic Publishers, 1993.

[8] J.-P. Merlet, Parallel robots, Kluwer Academic Publishers, 2000.

[9] E.C. Sherbrooke and N.M. Patrikalakis, "Computation of the solution of nonlinear polynomial systems," Computer Aided Geometric Design, Vol. 10, pp. 379405, 1993. 